\title{
ON WEIGHTED APPROXIMATION WITH JACOBI WEIGHTS *
}

\author{
K. Kopotun ${ }^{\dagger}$, D. Leviatan ${ }^{\ddagger}$ and I. A. Shevchuk ${ }^{\S}$
}

October 17, 2017

\begin{abstract}
We obtain matching direct and inverse theorems for the degree of weighted $L_{p^{-}}$ approximation by polynomials with the Jacobi weights $(1-x)^{\alpha}(1+x)^{\beta}$. Combined, the estimates yield a constructive characterization of various smoothness classes of functions via the degree of their approximation by algebraic polynomials.
\end{abstract}

\section{Introduction and main results}

In this paper, we are interested in weighted polynomial approximation with the Jacobi weights

$$
w_{\alpha, \beta}(x):=(1-x)^{\alpha}(1+x)^{\beta}, \quad \alpha, \beta \in J_{p}:= \begin{cases}(-1 / p, \infty), & \text { if } 0<p<\infty, \\ {[0, \infty),} & \text { if } p=\infty .\end{cases}
$$

Let $L_{p}^{\alpha, \beta}(I):=\left\{f \mid\left\|w_{\alpha, \beta} f\right\|_{L_{p}(I)}<\infty\right\}$, where $\|\cdot\|_{L_{p}(I)}$ is the usual $L_{p}$ (quasi)norm on the interval $I \subseteq[-1,1]$, and, for $f \in L_{p}^{\alpha, \beta}(I)$, denote by

$$
E_{n}(f, I)_{\alpha, \beta, p}:=\inf _{p_{n} \in \mathbb{P}_{n}}\left\|w_{\alpha, \beta}\left(f-p_{n}\right)\right\|_{L_{p}(I)}
$$

the error of best weighted approximation of $f$ by polynomials in $\mathbb{P}_{n}$, the set of algebraic polynomials of degree not more than $n-1$. For $I=[-1,1]$, we denote $\|\cdot\|_{p}:=\|\cdot\|_{L_{p}[-1,1]}, L_{p}^{\alpha, \beta}:=L_{p}^{\alpha, \beta}([-1,1]), E_{n}(f)_{\alpha, \beta, p}:=E_{n}(f,[-1,1])_{\alpha, \beta, p}$, etc.

Definition $1.1([13])$. For $r \in \mathbb{N}_{0}$ and $0<p \leq \infty$, denote $\mathbb{B}_{p}^{0}\left(w_{\alpha, \beta}\right):=L_{p}^{\alpha, \beta}$ and

$$
\mathbb{B}_{p}^{r}\left(w_{\alpha, \beta}\right):=\left\{f \mid f^{(r-1)} \in A C_{l o c}(-1,1) \quad \text { and } \quad \varphi^{r} f^{(r)} \in L_{p}^{\alpha, \beta}\right\}, \quad r \geq 1,
$$

where $\varphi(x):=\sqrt{1-x^{2}}$ and $A C_{l o c}(-1,1)$ denotes the set of functions which are locally absolutely continuous in $(-1,1)$.

*AMS classification: 41A10, 41A17, 41A25. Keywords and phrases: Approximation by polynomials in weighted $L_{p}$-norms, Degree of approximation, direct and inverse theorems, Jacobi weights, moduli of smoothness, characterization of smoothness classes.

${ }^{\dagger}$ Department of Mathematics, University of Manitoba, Winnipeg, Manitoba, R3T 2N2, Canada (kirill.kopotun@umanitoba.ca). Supported by NSERC of Canada.

${ }^{\ddagger}$ Raymond and Beverly Sackler School of Mathematical Sciences, Tel Aviv University, Tel Aviv 69978, Israel (leviatan@post.tau.ac.il).

${ }^{\S}$ Faculty of Mechanics and Mathematics, National Taras Shevchenko University of Kyiv, 01033 Kyiv, Ukraine (shevchuk@univ.kiev.ua). 
As is common when dealing with $L_{p}$ spaces, we will not distinguish between a function in $\mathbb{B}_{p}^{r}\left(w_{\alpha, \beta}\right)$ and all functions which are equivalent to it in $L_{p}^{\alpha, \beta}$.

maindefinition

wkrdefinition

$$
\omega_{k, r}^{\varphi}\left(f^{(r)}, t\right)_{\alpha, \beta, p}:=\sup _{0 \leq h \leq t}\left\|\mathcal{W}_{k h}^{r / 2+\alpha, r / 2+\beta}(\cdot) \Delta_{h \varphi(\cdot)}^{k}\left(f^{(r)}, \cdot\right)\right\|_{p},
$$

where

$$
\mathcal{W}_{\delta}^{\xi, \zeta}(x):=(1-x-\delta \varphi(x) / 2)^{\xi}(1+x-\delta \varphi(x) / 2)^{\zeta},
$$

and

$$
\Delta_{h}^{k}(f, x):= \begin{cases}\sum_{i=0}^{k}\left(\begin{array}{c}
k \\
i
\end{array}\right)(-1)^{k-i} f\left(x-\frac{k h}{2}+i h\right), & \text { if }\left[x-\frac{k h}{2}, x+\frac{k h}{2}\right] \subseteq[-1,1] \\
0, & \text { otherwise }\end{cases}
$$

is the $k$ th symmetric difference.

For $\delta>0$, denote (see [12])

$$
\mathfrak{D}_{\delta}:=\{x|1-\delta \varphi(x) / 2 \geq| x \mid\} \backslash\{ \pm 1\}=[-1+\mu(\delta), 1-\mu(\delta)],
$$

where

$$
\mu(\delta):=2 \delta^{2} /\left(4+\delta^{2}\right) .
$$

We note that $\mathfrak{D}_{\delta_{1}} \subset \mathfrak{D}_{\delta_{2}}$ if $\delta_{2}<\delta_{1} \leq 2$, and that $\mathfrak{D}_{\delta}=\emptyset$ if $\delta>2$. Also, since $\Delta_{h \varphi(x)}^{k}(f, x)=0$ if $x \notin \mathfrak{D}_{k h}$,

dom

$$
\omega_{k, r}^{\varphi}\left(f^{(r)}, t\right)_{\alpha, \beta, p}=\sup _{0<h \leq t}\left\|\mathcal{W}_{k h}^{r / 2+\alpha, r / 2+\beta}(\cdot) \Delta_{h \varphi(\cdot)}^{k}\left(f^{(r)}, \cdot\right)\right\|_{L_{p}\left(\mathfrak{D}_{k h}\right)} .
$$

In particular, $\omega_{k, r}^{\varphi}\left(f^{(r)}, t\right)_{\alpha, \beta, p}=\omega_{k, r}^{\varphi}\left(f^{(r)}, 2 / k\right)_{\alpha, \beta, p}$, for all $t \geq 2 / k$.

Following [13] we also define the weighted averaged moduli.

Definition 1.3 ([13]). For $k \in \mathbb{N}, r \in \mathbb{N}_{0}$ and $f \in \mathbb{B}_{p}^{r}\left(w_{\alpha, \beta}\right), 0<p<\infty$, the $k$ th weighted averaged modulus of smoothness of $f$ is defined as

$$
\omega_{k, r}^{* \varphi}\left(f^{(r)}, t\right)_{\alpha, \beta, p}:=\left(\frac{1}{t} \int_{0}^{t} \int_{\mathfrak{D}_{k \tau}}\left|\mathcal{W}_{k \tau}^{r / 2+\alpha, r / 2+\beta}(x) \Delta_{\tau \varphi(x)}^{k}\left(f^{(r)}, x\right)\right|^{p} d x d \tau\right)^{1 / p} .
$$

If $p=\infty$ and $f \in \mathbb{B}_{\infty}^{r}\left(w_{\alpha, \beta}\right)$, we write

$$
\omega_{k, r}^{* \varphi}\left(f^{(r)}, t\right)_{\alpha, \beta, \infty}:=\omega_{k, r}^{\varphi}\left(f^{(r)}, t\right)_{\alpha, \beta, \infty} .
$$

Clearly,

$$
\omega_{k, r}^{* \varphi}\left(f^{(r)}, t\right)_{\alpha, \beta, p} \leq \omega_{k, r}^{\varphi}\left(f^{(r)}, t\right)_{\alpha, \beta, p}, \quad t>0 .
$$

Moreover, it was proved in [13] that if $r / 2+\alpha, r / 2+\beta \geq 0$, then the weighted moduli and the weighted averaged moduli are equivalent.

Throughout this paper, all constants $c$ may depend only on $k, r, p, \alpha$ and $\beta$, unless a specific dependence on an additional parameter is mentioned.

We have the following direct (Jackson-type) theorem.

direct Theorem 1.4. Let $k \in \mathbb{N}, 0<p \leq \infty, \alpha \geq 0$ and $\beta \geq 0$. If $f \in L_{p}^{\alpha, \beta}$, then

$$
E_{n}(f)_{\alpha, \beta, p} \leq c \omega_{k, 0}^{\varphi}(f, 1 / n)_{\alpha, \beta, p}, \quad n \geq k .
$$


It follows from [13, Lemma 1.11] that, if $k \in \mathbb{N}, r \in \mathbb{N}_{0}, r / 2+\alpha \geq 0, r / 2+\beta \geq 0$, $1 \leq p \leq \infty$ and $f \in \mathbb{B}_{p}^{r+1}\left(w_{\alpha, \beta}\right)$, then

$$
\omega_{k+1, r}^{\varphi}\left(f^{(r)}, t\right)_{\alpha, \beta, p} \leq c t \omega_{k, r+1}^{\varphi}\left(f^{(r+1)}, t\right)_{\alpha, \beta, p}, \quad t>0 .
$$

Hence, (1.5) implies that, for $f \in B_{p}^{r}\left(w_{\alpha, \beta}\right), 1 \leq p \leq \infty$,

$$
E_{n}(f)_{\alpha, \beta, p} \leq c \omega_{k+r, 0}^{\varphi}(f, 1 / n)_{\alpha, \beta, p} \leq c t^{r} \omega_{k, r}^{\varphi}\left(f^{(r)}, 1 / n\right)_{\alpha, \beta, p}, \quad n \geq k+r,
$$

provided $\alpha, \beta \geq 0$. We strengthen this result by showing that the last estimate is, in fact, valid for all $\alpha, \beta \geq-r / 2$. Namely,

thm2direct

Theorem 1.5. Let $k \in \mathbb{N}, r \in \mathbb{N}_{0}, 1 \leq p \leq \infty$, and $\alpha, \beta \in J_{p}$ be such that $r / 2+\alpha \geq 0$ and $r / 2+\beta \geq 0$. If $f \in \mathbb{B}_{p}^{r}\left(w_{\alpha, \beta}\right)$, then

thm2dir

$$
E_{n}(f)_{w_{\alpha, \beta}, p} \leq \frac{c}{n^{r}} \omega_{k, r}^{\varphi}\left(f^{(r)}, 1 / n\right)_{\alpha, \beta, p}, \quad n \geq k+r .
$$

We remark that Theorem 1.5 is not valid if $r \geq 1$ and $0<p<1$ (one can show this using the same construction that was used in the proof of [8, Theorem 3 and Corollary 4]).

Next, we have the following inverse result in the case $1 \leq p \leq \infty$.

inverse Theorem 1.6. Suppose that $r \in \mathbb{N}_{0}, 1 \leq p \leq \infty, \alpha, \beta \in J_{p}$ are such that $r / 2+\alpha \geq$ 0 and $r / 2+\beta \geq 0$, and $f \in L_{p}^{\alpha, \beta}$. If

$$
\sum_{n=1}^{\infty} r n^{r-1} E_{n}(f)_{w_{\alpha, \beta}, p}<+\infty
$$

(i.e., if $r=0$ then this condition is vacuous), then $f \in \mathbb{B}_{p}^{r}\left(w_{\alpha, \beta}\right)$, and for $k \in \mathbb{N}$ and $N \in \mathbb{N}$,

inverse1

$$
\begin{aligned}
\omega_{k, r}^{\varphi}\left(f^{(r)}, t\right)_{\alpha, \beta, p} \leq c & \sum_{n>\max \{N, 1 / t\}} r n^{r-1} E_{n}(f)_{w_{\alpha, \beta}, p} \\
& +c t^{k} \sum_{N \leq n \leq \max \{N, 1 / t\}} n^{k+r-1} E_{n}(f)_{w_{\alpha, \beta}, p} \\
& +c(N) t^{k} E_{k+r}(f)_{w_{\alpha, \beta}, p}, \quad t>0 .
\end{aligned}
$$

In particular, if $N \leq k+r$, then

$$
\begin{aligned}
\omega_{k, r}^{\varphi}\left(f^{(r)}, t\right)_{\alpha, \beta, p} \leq & c \sum_{n>\max \{N, 1 / t\}} r n^{r-1} E_{n}(f)_{w_{\alpha, \beta}, p} \\
& +c t^{k} \sum_{N \leq n \leq \max \{N, 1 / t\}} n^{k+r-1} E_{n}(f)_{w_{\alpha, \beta}, p}, t>0 .
\end{aligned}
$$

rem Remark 1.7. (i) Note that the first term in (1.8) disappears if $r=0$.

(ii) If $\alpha=\beta=0$, Theorem 1.6 was proved in [12].

(iii) The case $\alpha, \beta \geq 0, N=1$ and $r=0$ follows from [5, Theorem 8.2.4] by virtue of (4.2).

Denote by $\Phi$ the set of nondecreasing functions $\phi:[0, \infty) \rightarrow[0, \infty)$, satisfying $\lim _{t \rightarrow 0^{+}} \phi(t)=0$. The following is an immediate corollary of Theorem 1.6 (in fact, it is a restatement of Theorem 1.6 in terms of $\phi$ ). 
Corollary 1.8. Suppose that $r \in \mathbb{N}_{0}, N \in \mathbb{N}, 1 \leq p \leq \infty, \alpha, \beta \in J_{p}$ are such that $r / 2+\alpha \geq 0$ and $r / 2+\beta \geq 0$, and $\phi \in \Phi$ is such that

$$
\int_{0}^{1} \frac{r \phi(u)}{u^{r+1}} d u<+\infty
$$

(i.e., if $r=0$ then this condition is vacuous). Then, if $f \in L_{p}^{\alpha, \beta}$ is such that

$$
E_{n}(f)_{w_{\alpha, \beta}, p} \leq \phi\left(\frac{1}{n+1}\right), \text { for all } n \geq N
$$

then $f \in \mathbb{B}_{p}^{r}\left(w_{\alpha, \beta}\right)$, and for $k \in \mathbb{N}$ and $0<t \leq 1 / 2$,

$$
\omega_{k, r}^{\varphi}\left(f^{(r)}, t\right)_{\alpha, \beta, p} \leq c \int_{0}^{t} \frac{r \phi(u)}{u^{r+1}} d u+c t^{k} \int_{t}^{1} \frac{\phi(u)}{u^{k+r+1}} d u+c(N) t^{k} E_{k+r}(f)_{w_{\alpha, \beta}, p} .
$$

In particular, if $N \leq k+r$, then

$$
\omega_{k, r}^{\varphi}\left(f^{(r)}, t\right)_{\alpha, \beta, p} \leq c \int_{0}^{t} \frac{r \phi(u)}{u^{r+1}} d u+c t^{k} \int_{t}^{1} \frac{\phi(u)}{u^{k+r+1}} d u .
$$

Remark 1.9. We take this opportunity to correct an inadvertent misprint in three of our earlier papers where the inverse theorems of this type were proved in the case $\alpha=\beta=0$. Namely, the inequality $E_{n}(f)_{p} \leq \phi(1 / n)$ in [10, Theorem 3.2] (the case $p=\infty$ ), and in [12, Theorem 9.1] and [11, Theorem $I_{r}$ ] (the case $1 \leq p \leq \infty)$, should be replaced by $E_{n}(f)_{p} \leq \phi(1 /(n+1))$. Otherwise, the last estimates in these results are not justified/valid if $N=1, k=1$ and $r=0$ since $E_{k+r}(f)_{p}=E_{1}(f)_{p} \leq \phi(1)$ cannot be estimated above by $\int_{t}^{1} \phi(u) u^{-2} d u$ without any extra assumptions on the function $\phi$.

It immediately follows from Theorem 1.4 that if $\alpha, \beta \in J_{p}, r / 2+\alpha \geq 0$, $r / 2+\beta \geq 0$ and $\omega_{k, r}^{\varphi}\left(f^{(r)}, t\right)_{\alpha, \beta, p} \leq t^{\gamma}$, then $E_{n}(f)_{w_{\alpha, \beta}, p} \leq c n^{-r-\gamma}$. Conversely, an immediate consequence of Theorem 1.6 (Corollary 1.8) is the following result which, for $\alpha, \beta \geq 0$, was proved by a different method in [11, Theorem 5.3].

Corollary 1.10. Suppose that $r \in \mathbb{N}_{0}, N \in \mathbb{N}, 1 \leq p \leq \infty$, and $\alpha, \beta \in J_{p}$ are such that $r / 2+\alpha \geq 0$ and $r / 2+\beta \geq 0$. If $f \in L_{p}^{\alpha, \beta}$ is such that, for some $N \in \mathbb{N}$ and $r<\gamma<k+r$,

$$
E_{n}(f)_{w_{\alpha, \beta}, p} \leq n^{-\gamma}, \quad n \geq N
$$

then $f \in \mathbb{B}_{p}^{r}\left(w_{\alpha, \beta}\right)$, and

$$
\omega_{k, r}^{\varphi}\left(f^{(r)}, t\right)_{\alpha, \beta, p} \leq c t^{\gamma-r}+c(N) t^{k} E_{k+r}(f)_{w_{\alpha, \beta}, p}, \quad t>0 .
$$

In particular, if $N \leq k+r$, then

$$
\omega_{k, r}^{\varphi}\left(f^{(r)}, t\right)_{\alpha, \beta, p} \leq c t^{\gamma-r}, \quad t>0 .
$$

Finally, we have the following inverse theorem for $0<p<1$ which is an immediate corollary of [9, Theorem 10.1] and [10, Lemma 4.5].

Theorem 1.11. Let $k \in \mathbb{N}, \alpha \geq 0, \beta \geq 0$ and $f \in L_{p}^{\alpha, \beta}, 0<p<1$. Then there exists a positive constant $\vartheta \leq 1$ depending only on $k, p, \alpha$ and $\beta$ such that, for any $n \in \mathbb{N}$,

$$
\omega_{k, 0}^{\varphi}\left(f, \vartheta n^{-1}\right)_{\alpha, \beta, p}^{p} \leq c n^{-k p} \sum_{m=1}^{n} m^{k p-1} E_{m}(f)_{w_{\alpha, \beta}, p}^{p} .
$$




\section{Auxiliary lemmas}

der

Lemma 2.1. Let $k \in \mathbb{N}, 0<\delta \leq 2$, and let $y:=y(x), y:[-1,1] \rightarrow \mathbb{R}$ be such that

$$
y(x)+\delta \varphi(y(x)) / 2=x, \quad x \in[-1,1] .
$$

Then,

(i) $y=y(x)$ is strictly increasing on $[-1,1]$, and $y^{\prime}(x) \leq 2, x \in[-1,1]$,

(ii) $y([-1+2 \mu(\delta), 1])=\mathfrak{D}_{\delta}$,

(iii) $y^{\prime}(x) \geq 2 / 3, x \in[-1+2 \mu(\delta), 1]$,

(iv) if $y_{\lambda}(x):=y(x)+\lambda \varphi(y(x))$, then $1 / 3 \leq y_{\lambda}^{\prime}(x) \leq 3$, for all $|\lambda| \leq \delta / 2$ and $x \in[-1+2 \mu(\delta), 1]$,

(v) for all $x \in[-1+2 \mu(\delta), 1]$,

$$
\mu(\delta)+2(1-x) / 3 \leq 1-y(x) \leq \mu(\delta)+2(1-x)
$$

and

$$
(1+x) / 2 \leq 1+y(x) \leq 1+x .
$$

Proof. Since $x \leq 1$, we have $y+\delta \varphi(y) / 2 \leq 1$ which can be rewritten as $\delta /(2 \varphi(y)) \leq$ $1 /(1+y)$, and so, if $y \geq 0$, then

$$
1-\frac{\delta y}{2 \varphi(y)} \geq \frac{1}{1+y} \geq \frac{1}{2}
$$

and, clearly, $1-\delta y /(2 \varphi(y)) \geq 1 / 2$ if $y<0$ as well.

Therefore, since

$$
\frac{d y}{d x}=\left(1-\frac{\delta y}{2 \varphi(y)}\right)^{-1},
$$

we immediately conclude that (ii) holds.

Now, since $y$ is nondecreasing, $y([-1+2 \mu(\delta), 1])=[y(-1+2 \mu(\delta)), y(1)]$, and (iii) follows because $y(1)=1-\mu(\delta)$ and $y(-1+2 \mu(\delta))=-1+\mu(\delta)$.

It follows from (iii) that, for $x \in[-1+2 \mu(\delta), 1]$, we have $y-\delta \varphi(y) / 2 \geq-1$, which can be rewritten as $\delta /(2 \varphi(y)) \leq 1 /(1-y)$, and so, if $y \leq 0$, then

$$
1-\frac{\delta y}{2 \varphi(y)} \leq \frac{1-2 y}{1-y} \leq \frac{3}{2}
$$

and, clearly, $1-\delta y /(2 \varphi(y)) \leq 3 / 2$ if $y>0$ as well. This implies (iii).

Now, it follows from the above estimates that $\delta /(2 \varphi(y)) \leq 1 /(1+|y|)$, for $x \in[-1+2 \mu(\delta), 1]$, which implies

$$
y_{\lambda}^{\prime}(x)=\left(1-\frac{\lambda y}{\varphi(y)}\right) y^{\prime}(x) \leq 2\left(1+\frac{\delta|y|}{2 \varphi(y)}\right) \leq \frac{2+4|y|}{1+|y|} \leq 3
$$

and

$$
y_{\lambda}^{\prime}(x) \geq \frac{2}{3}\left(1-\frac{\delta|y|}{2 \varphi(y)}\right) \geq \frac{2}{3(1+|y|)} \geq \frac{1}{3},
$$

and so (iv) is verified.

Now, by

$$
\frac{d y}{d x}(\xi)=\frac{y(1)-y(x)}{1-x}=\frac{1-\mu(\delta)-y(x)}{1-x}
$$


(ii) and (iii) imply, for $x \in[-1+2 \mu(\delta), 1]$,

$$
\mu(\delta)+2(1-x) / 3 \leq 1-y(x) \leq \mu(\delta)+2(1-x),
$$

which is (2.1). Finally, the second inequality in (2.2) is obvious, and the first one immediately follows from (iii) which implies

$$
1+x=1+y+\delta \varphi(y) / 2 \leq 2(1+y)
$$

Thus, ( $(\mathbb{\nabla})$ is verified.

\section{$3 \quad$ Whitney-type estimates}

In this section, we prove Whitney-type estimates, which we feel are of independent interest, and which we need in order to prove the direct (Jackson-type) theorem (Theorem 1.4) for small $n$.

Recall that the celebrated Whitney inequalities for the ordinary moduli of smoothness were first proved by Whitney [19] for functions in $C[a, b]$. Later Brudnyi [1] extended the inequalities to $L_{p}[a, b], 1 \leq p<\infty$ and, finally, Storozhenko [18] proved the inequalities for $L_{p}[a, b], 0<p<1$.

Theorem 3.1. Let $k \in \mathbb{N}, \alpha \geq 0, \beta \geq 0,0<p \leq \infty, f \in L_{p}^{\alpha, \beta}, 0<h \leq 2$ and $x_{0} \in \mathfrak{D}_{h}$. Then, for any $\theta \in(0,1]$, we have

$$
E_{k}\left(f,\left[x_{0}-h \varphi\left(x_{0}\right) / 2, x_{0}+h \varphi\left(x_{0}\right) / 2\right]\right)_{w_{\alpha, \beta}, p} \leq c \omega_{k, 0}^{* \varphi}(f, \theta h)_{\alpha, \beta, p} \leq c \omega_{k, 0}^{\varphi}(f, \theta h)_{\alpha, \beta, p},
$$

where $c$ depends only on $k, \alpha, \beta, p$ and $\theta$.

Choosing $x_{0}=0$ and $h=2$ in Theorem 3.1 (and replacing $2 \theta$ by $\theta$ ) we immediately get the following corollary.

whcor Corollary 3.2. Let $k \in \mathbb{N}, \alpha \geq 0, \beta \geq 0,0<p \leq \infty$ and $f \in L_{p}^{\alpha, \beta}$. Then, for any $\theta \in(0,1]$, we have

ineq:regwh

$$
E_{k}(f)_{w_{\alpha, \beta}, p} \leq c \omega_{k, 0}^{* \varphi}(f, \theta)_{\alpha, \beta, p} \leq c \omega_{k, 0}^{\varphi}(f, \theta)_{\alpha, \beta, p},
$$

where $c$ depends only on $k, \alpha, \beta, p$ and $\theta$.

Also, if $x_{0} \pm h \varphi\left(x_{0}\right) / 2= \pm 1$, Theorem 3.1 immediately gives the following result (by letting $h:=t \sqrt{4 A /\left(4-A t^{2}\right)}, x_{0}:= \pm(1-\mu(h)), \theta:=\min \{1,1 / \sqrt{2 A}\}$, and using monotonicity of the moduli with respect to $t)$.

corwh Corollary 3.3. Let $k \in \mathbb{N}, \alpha \geq 0, \beta \geq 0, A>0,0<p \leq \infty$ and $f \in L_{p}^{\alpha, \beta}$. Then, for any $0<t \leq \sqrt{2 / A}$, we have

$$
E_{k}\left(f,\left[1-A t^{2}, 1\right]\right)_{w_{\alpha, \beta}, p} \leq c \omega_{k, 0}^{* \varphi}(f, t)_{\alpha, \beta, p} \leq c \omega_{k, 0}^{\varphi}(f, t)_{\alpha, \beta, p}
$$

and

$$
E_{k}\left(f,\left[-1,-1+A t^{2}\right]\right)_{w_{\alpha, \beta}, p} \leq c \omega_{k, 0}^{* \varphi}(f, t)_{\alpha, \beta, p} \leq c \omega_{k, 0}^{\varphi}(f, t)_{\alpha, \beta, p},
$$

where $c$ depends on $k, p, \alpha, \beta$ and $A$. 
Proof of Theorem 3.1. Theorem 3.1 follows from the classical (non-weighted) Whitney's inequality (see [4, Theorem 6.4.2 and Theorem 12.5.5]), which readily implies (see e.g. [16, Sections 3.1 and 7.1]), for each interval $J \subset[-1,1]$, the existence of a polynomial $p_{k} \in \mathbb{P}_{k}$, such that

initial

$$
\left\|f-p_{k}\right\|_{L_{p}(J)} \leq c \omega_{k}(f,|J| ; J)_{p} \leq c \frac{|J|^{k-1+1 / p_{1}}}{\delta^{k-1+1 / p_{1}}} \omega_{k}(f, \delta ; J)_{p}, \quad 0<\delta \leq|J|,
$$

where $|J|$ is the length of of the interval $J$ and $p_{1}:=\min \{1, p\}$.

In order to prove Theorem 3.1, we assume, without loss of generality, that $x_{0} \geq 0$, and denote

$$
[a, b]:=\left[x_{0}-h \varphi\left(x_{0}\right) / 2, x_{0}+h \varphi\left(x_{0}\right) / 2\right], \quad W_{p}:=\omega_{k, 0}^{* \varphi}(f, \theta h)_{\alpha, \beta, p},
$$

Note that

$$
1-x \leq 2\left(1-x_{0}\right) \text { and } 1+x \leq 2\left(1+x_{0}\right), \quad x \in[a, b],
$$

since $x_{0}$ is the middle of $[a, b]$, and so

$$
\varphi(b) \leq \varphi(x) \leq 2 \varphi\left(x_{0}\right), \quad \text { for all } \quad x \in[a, b],
$$

where the first inequality is valid since $|x| \leq|b|$ (because $x_{0}$ is assumed to be nonnegative).

We will consider two cases: (i) $\varphi\left(x_{0}\right) \leq 2 \varphi(b)$ and (ii) $\varphi\left(x_{0}\right)>2 \varphi(b)$.

Case (i): $\varphi\left(x_{0}\right) \leq 2 \varphi(b), x \in[a, b]$.

Then, for all $x \in[a, b]$,

$$
1-x_{0} \leq \varphi^{2}\left(x_{0}\right) \leq 4 \varphi^{2}(b) \leq 4 \varphi^{2}(x)<8(1-x)
$$

and

$$
1+x_{0}=\frac{\varphi^{2}\left(x_{0}\right)}{1-x_{0}} \leq \frac{4 \varphi^{2}(b)}{1-x_{0}} \leq \frac{8 \varphi^{2}(x)}{1-x}=8(1+x) .
$$

Now, let $J:=[a, b]$ and $\delta:=\theta h \varphi(b)$, and note that

$$
\frac{\theta}{2}|J|=\frac{\theta}{2} h \varphi\left(x_{0}\right) \leq \delta \leq \theta h \varphi\left(x_{0}\right) \leq|J| .
$$

So, for $p=\infty$, we have

$$
\begin{aligned}
\omega_{k}(f, \delta ; J)_{\infty} & =\sup _{0<s \leq \delta} \sup _{x \in J}\left|\Delta_{s}^{k}(f, x ; J)\right|=\sup _{0<\tau \leq \delta / \varphi(b)} \sup _{x \in J}\left|\Delta_{\tau \varphi(b)}^{k}(f, x ; J)\right| \\
& =\sup _{0<\tau \leq \theta h} \sup _{x \in J}\left|\Delta_{\tau \varphi(b)}^{k}(f, x ; J)\right| \leq \sup _{0<\tau \leq \theta h} \sup _{x \in J}\left|\Delta_{\tau \varphi(x)}^{k}(f, x ; J)\right| \\
& \leq c w_{\alpha, \beta}^{-1}\left(x_{0}\right) \sup _{0<\tau \leq \theta h x \in J} \sup _{x \in J}\left|\mathcal{W}_{k \tau}^{\alpha, \beta}(x) \Delta_{\tau \varphi(x)}^{k}(f, x ; J)\right| \\
& =c w_{\alpha, \beta}^{-1}\left(x_{0}\right) W_{\infty},
\end{aligned}
$$

where in the last inequality we used the fact that the estimates (3.5) and (3.6) imply that $w_{\alpha, \beta}(x) \leq c \mathcal{W}_{k \tau}^{\alpha, \beta}(x)$, for all $x$ such that $x \pm k \tau \varphi(x) / 2 \in J$.

If $p<\infty$, then it is well known (see e.g. [16, Lemma 7.2]) that

$$
\omega_{k}(f, t ; J)_{p}^{p} \leq c \frac{1}{t} \int_{0}^{t} \int_{J}\left|\Delta_{s}^{k}(f, x ; J)\right|^{p} d x d s, \quad 0<t \leq|J| / k .
$$


Hence, using (3.7) and (3.4) we have

$$
\begin{aligned}
c \delta \omega_{k}(f, \delta ; J)_{p}^{p} & \leq \int_{J} \int_{0}^{\delta}\left|\Delta_{s}^{k}(f, x, J)\right|^{p} d s d x \\
& =\int_{J} \int_{0}^{\delta / \varphi(x)} \varphi(x)\left|\Delta_{\tau \varphi(x)}^{k}(f, x, J)\right|^{p} d \tau d x \\
& \leq \int_{J} \int_{0}^{\theta h} \varphi(x)\left|\Delta_{\tau \varphi(x)}^{k}(f, x, J)\right|^{p} d \tau d x \\
& \leq c w_{\alpha, \beta}^{-p}\left(x_{0}\right) \varphi(b) \int_{J} \int_{0}^{\theta h}\left|\mathcal{W}_{k \tau}^{\alpha, \beta}(x) \Delta_{\tau \varphi(x)}^{k}(f, x, J)\right|^{p} d \tau d x \\
& \leq c w_{\alpha, \beta}^{-p}\left(x_{0}\right) \varphi(b) \int_{0}^{\theta h} \int_{\mathfrak{D}_{k \tau}}\left|\mathcal{W}_{k \tau}^{\alpha, \beta}(x) \Delta_{\tau \varphi(x)}^{k}(f, x)\right|^{p} d x d \tau \\
& =c w_{\alpha, \beta}^{-p}\left(x_{0}\right) \theta h \varphi(b) W_{p}^{p} .
\end{aligned}
$$

Thus, for all $0<p \leq \infty$, we have

$$
\omega_{k}(f, \delta ; J)_{p} \leq c w_{\alpha, \beta}^{-1}\left(x_{0}\right) W_{p},
$$

which, by virtue of (3.3), yields

$$
\left\|w_{\alpha, \beta}\left(f-p_{k}\right)\right\|_{L_{p}(J)} \leq c w_{\alpha, \beta}\left(x_{0}\right)\left\|f-p_{k}\right\|_{L_{p}(J)} \leq c w_{\alpha, \beta}\left(x_{0}\right) \omega_{k}(f, \delta ; J)_{p} \leq c W_{p},
$$

and so the proof is complete in Case (i).

Case (ii): $\varphi\left(x_{0}\right)>2 \varphi(b)$.

We first note that, in this case, it suffices to assume that $b=1$. Indeed, suppose that the theorem is proved for all $\hat{x}_{0}$ and $\hat{h}$ such that $\hat{x}_{0}+\hat{h} \varphi\left(\hat{x}_{0}\right) / 2=1$, and let $x_{0}$ and $h$ be such that $\varphi\left(x_{0}\right)>2 \varphi(b)$ (recall that $b=x_{0}+h \varphi\left(x_{0}\right) / 2$ ). We let $\hat{x}_{0}:=x_{0}, \hat{h}:=2\left(1-x_{0}\right) / \varphi\left(x_{0}\right)$ and note that $x_{0}+\hat{h} \varphi\left(x_{0}\right) / 2=1$. Now, since

$$
1-x_{0}=\frac{\varphi^{2}\left(x_{0}\right)}{1+x_{0}}>\frac{4 \varphi^{2}(b)}{1+x_{0}}=4(1-b) \frac{1+b}{1+x_{0}} \geq 4(1-b),
$$

we have

$$
h \varphi\left(x_{0}\right)=2\left(b-x_{0}\right)=2\left(1-x_{0}\right)-2(1-b)>3\left(1-x_{0}\right) / 2 .
$$

Therefore, $h \leq \hat{h} \leq 4 h / 3$, and so

$$
\begin{aligned}
& E_{k}\left(f,\left[x_{0}-h \varphi\left(x_{0}\right) / 2, x_{0}+h \varphi\left(x_{0}\right) / 2\right]\right)_{w_{\alpha, \beta}, p} \\
& \quad \leq E_{k}\left(f,\left[x_{0}-\hat{h} \varphi\left(x_{0}\right) / 2, x_{0}+\hat{h} \varphi\left(x_{0}\right) / 2\right]\right)_{w_{\alpha, \beta}, p} \\
& \quad \leq c \omega_{k, 0}^{* \varphi}\left(f, \theta_{1} \hat{h}\right)_{\alpha, \beta, p} \leq c W_{p},
\end{aligned}
$$

where $\theta_{1}:=3 \theta / 4$.

Hence, for the rest of this proof, we assume that $b=1$. Note that

$$
b-a=h \varphi\left(x_{0}\right)=2\left(1-x_{0}\right)=2 \mu(h) \in\left[h^{2} / 2, h^{2}\right] .
$$

Define

$$
\tilde{h}:=\frac{\theta h}{10 k}, \quad \tilde{b}:=1-\tilde{h}^{2} \quad \text { and } \quad J:=[a, b] \cap[-\tilde{b}, \tilde{b}] .
$$


Then $x_{0} \in J$, and, for all $x \in J$, we have

$$
\frac{1-x_{0}}{1-x} \leq \frac{\mu(h)}{\tilde{h}^{2}}<c, \quad \frac{1+x_{0}}{1+x} \leq \frac{2}{\max \left\{\tilde{h}^{2}, 1+a\right\}} \leq \frac{c}{\max \left\{h^{2}, 4-h^{2}\right\}}<c
$$

and

$$
\varphi(\tilde{b}) \leq \varphi(x) \leq 2 \varphi\left(x_{0}\right) \leq c \varphi(\tilde{b})
$$

We now let $\delta:=\theta h \varphi(\tilde{b})$, note that

$$
c|J| \leq c(b-a) \leq \delta \leq b-a \leq c|J|,
$$

and conclude using the same argument that was used in Case (i) that there is a polynomial $p_{k} \in \mathbb{P}_{k}$, such that

$$
\left\|w_{\alpha, \beta}\left(f-p_{k}\right)\right\|_{L_{p}(J)} \leq c W_{p} .
$$

So, to finish the proof in Case (ii) we have to show that, for the function $g:=f-p_{k}$, the inequalities

$$
\left\|w_{\alpha, \beta} g\right\|_{L_{p}[\tilde{b}, 1]} \leq c W_{p}
$$

and, if $a<-\tilde{b}$,

$$
\left\|w_{\alpha, \beta} g\right\|_{L_{p}[a,-\tilde{b}]} \leq c W_{p} .
$$

hold. We prove (3.11), the proof of (3.12) being similar.

To this end let $t \in[2 \tilde{h} / \sqrt{k}, 4 \tilde{h} / \sqrt{k}]$ be fixed for now, and denote by $y=y(x)$ and $y_{i}=y_{i}(x), 1 \leq i \leq k$, the functions such that

$y(x)+k t \varphi(y(x)) / 2=x \quad$ and $\quad y_{i}(x):=x-i t \varphi(y(x))=y(x)+(k / 2-i) t \varphi(y(x))$.

We now note that $[\tilde{b}, 1] \subset[-1+2 \mu(k t), 1]$, since

$$
-1+2 \mu(k t) \leq-1+k^{2} t^{2} \leq-1+16 k \tilde{h}^{2} \leq 1-\tilde{h}^{2}=\tilde{b}
$$

and so Lemma 2.1 with $\delta=k t$ implies that, for all $x \in[\tilde{b}, 1], 2 / 3 \leq y^{\prime}(x) \leq 2$, $1 / 3 \leq y_{i}^{\prime}(x) \leq 3$, and

$$
\varphi^{2}(y(x)) \leq 2\left(\mu(k t)+2 \tilde{h}^{2}\right) \leq k^{2} t^{2}+4 \tilde{h}^{2} \leq 25 k \tilde{h}^{2} .
$$

Additionally, note that

$$
y_{i}(x) \in J, \quad x \in[\tilde{b}, 1] \quad \text { and } \quad 1 \leq i \leq k .
$$

Indeed, since $y(1)=1-\mu(k t)$, we have, for $x \in[\tilde{b}, 1]$,

$$
y_{i}(x) \leq y_{1}(x) \leq y_{1}(1)=1-t \varphi(y(1))=1-2 \mu(k t) / k \leq 1-k t^{2} / 2 \leq 1-2 \tilde{h}^{2}<\tilde{b},
$$

and, using (3.13) and (3.8),

$$
\begin{aligned}
y_{i}(x) & \geq y_{k}(x) \geq y_{k}(\tilde{b})=\tilde{b}-k t \varphi(y(\tilde{b})) \geq 1-\tilde{h}^{2}-5 k^{3 / 2} t \tilde{h} \geq 1-21 k \tilde{h}^{2} \\
& \geq \max \left\{-1+\tilde{h}^{2}, a\right\},
\end{aligned}
$$

which yields (3.14). Note also that the above implies that $1+y(x) \geq 3 k t \varphi(y(x)) / 2$, for $x \in[\tilde{b}, 1]$. 
Hence,

$$
w_{\alpha, \beta}(x)=w_{\alpha, \beta}(y(x)+k t \varphi(y(x)) / 2) \leq 2^{\beta} w_{\alpha, \beta}\left(y_{i}(x)\right), \quad x \in[\tilde{b}, 1],
$$

and using (3.14) and (3.10) we get, for $0<p<\infty$,

$$
\begin{aligned}
\left\|w_{\alpha, \beta} g\left(y_{i}\right)\right\|_{L_{p}[\tilde{b}, 1]} & \leq 2^{\beta}\left\|w_{\alpha, \beta}\left(y_{i}\right) g\left(y_{i}\right)\right\|_{L_{p}[\tilde{b}, 1]} \leq c\left\|w_{\alpha, \beta}\left(y_{i}\right) g\left(y_{i}\right)\left(y_{i}^{\prime}\right)^{1 / p}\right\|_{L_{p}[\tilde{b}, 1]} \\
& \leq c\left\|w_{\alpha, \beta} g\right\|_{L_{p}(J)} \leq c W_{p} .
\end{aligned}
$$

If $p=\infty$, then similar (and, in fact, simpler) arguments yield

$$
\left\|w_{\alpha, \beta} g\left(y_{i}\right)\right\|_{L_{\infty}[\tilde{b}, 1]} \leq c W_{\infty}, \quad 1 \leq i \leq k .
$$

Now, for $x \in[\tilde{b}, 1]$,

$$
\begin{aligned}
g(x) & =\Delta_{t \varphi(y(x))}^{k}(g, y(x))-\sum_{i=0}^{k-1}(-1)^{k-i}\left(\begin{array}{c}
k \\
i
\end{array}\right) g\left(y(x)+\left(i-\frac{k}{2}\right) t \varphi(y(x))\right) \\
& =\Delta_{t \varphi(y(x))}^{k}(g, y(x))-\sum_{i=1}^{k}(-1)^{i}\left(\begin{array}{c}
k \\
i
\end{array}\right) g\left(y_{i}(x)\right),
\end{aligned}
$$

and so

$$
\begin{aligned}
\left\|w_{\alpha, \beta} g\right\|_{L_{p}[\tilde{b}, 1]} & \leq c\left\|w_{\alpha, \beta} \Delta_{t \varphi(y)}^{k}(g, y)\right\|_{L_{p}[\tilde{b}, 1]}+c \sum_{i=1}^{k}\left(\begin{array}{c}
k \\
i
\end{array}\right)\left\|w_{\alpha, \beta} g\left(y_{i}\right)\right\|_{L_{p}[\tilde{b}, 1]} \\
& \leq c\left\|w_{\alpha, \beta} \Delta_{t \varphi(y)}^{k}(g, y)\right\|_{L_{p}[\tilde{b}, 1]}+c W_{p} \\
& \leq c\left\|\mathcal{W}_{t k}^{\alpha, \beta}(y) \Delta_{t \varphi(y)}^{k}(g, y)\right\|_{L_{p}[\tilde{b}, 1]}+c W_{p} \\
& \leq c\left\|\mathcal{W}_{t k}^{\alpha, \beta} \Delta_{t \varphi}^{k}(g, \cdot)\right\|_{L_{p}\left(\mathfrak{D}_{k t}\right)}+c W_{p} .
\end{aligned}
$$

This completes the proof in the case $p=\infty$. If $p<\infty$, then integrating with respect to $t$ over $[2 \tilde{h} / \sqrt{k}, 4 \tilde{h} / \sqrt{k}]$ we get

$$
\left\|w_{\alpha, \beta} g\right\|_{L_{p}[\tilde{b}, 1]}^{p} \leq \frac{c}{\tilde{h}} \int_{2 \tilde{h} / \sqrt{k}}^{4 \tilde{h} / \sqrt{k}}\left\|\mathcal{W}_{t k}^{\alpha, \beta} \Delta_{t \varphi}^{k}(g, \cdot)\right\|_{L_{p}\left(\mathfrak{D}_{k t}\right)}^{p} d t+c W_{p}^{p} \leq c W_{p}^{p} .
$$

The proof is now complete.

We now prove a Whitney-type result for functions from $f \in \mathbb{B}_{p}^{r}\left(w_{\alpha, \beta}\right), r \in \mathbb{N}$.

whitneythm

Theorem 3.4. Let $k \in \mathbb{N}, r \in \mathbb{N}, 1 \leq p \leq \infty$, and let $\alpha, \beta \in J_{p}$ be such that $r / 2+\alpha \geq 0$ and $r / 2+\beta \geq 0$. If $f \in \mathbb{B}_{p}^{r}\left(w_{\alpha, \beta}\right)$, then for any $\theta \in(0,1]$,

whitneyineq

$$
E_{k+r}(f)_{w_{\alpha, \beta}, p} \leq c \omega_{k, r}^{\varphi}\left(f^{(r)}, \theta\right)_{\alpha, \beta, p}
$$

Proof. Note that $f \in \mathbb{B}_{p}^{r}\left(w_{\alpha, \beta}\right)$ implies that $f^{(r)} \in L_{p}^{r / 2+\alpha, r / 2+\beta}$, and so it follows from (3.1) that

$$
E_{k}\left(f^{(r)}\right)_{w_{r / 2+\alpha, r / 2+\beta, p}} \leq c \omega_{k, 0}^{\varphi}\left(f^{(r)}, \theta\right)_{r / 2+\alpha, r / 2+\beta, p}=c W_{r, p}
$$

where $W_{r, p}:=\omega_{k, r}^{\varphi}\left(f^{(r)}, \theta\right)_{\alpha, \beta, p}$. 
Let $\tilde{P}_{k} \in \mathbb{P}_{k}$ be a polynomial such that

$$
\left\|w_{r / 2+\alpha, r / 2+\beta}\left(f^{(r)}-\tilde{P}_{k}\right)\right\|_{p}<c W_{r, p}
$$

and define $P_{k+r} \in \mathbb{P}_{k+r}$ by

$$
P_{k+r}(x):=f(0)+\frac{f^{\prime}(0)}{1 !} x+\cdots+\frac{f^{(r-1)}(0)}{(r-1) !} x^{r-1}+\frac{1}{(r-1) !} \int_{0}^{x}(x-u)^{r-1} \tilde{P}_{k}(u) d u .
$$

Assuming that $x \geq 0$ (for $x<0$ all estimates are analogous), we have by Hölder's inequality

$$
\begin{aligned}
& (r-1) !\left|f(x)-P_{k+r}(x)\right| \\
& \quad \leq \int_{0}^{x}(x-u)^{r-1}\left|f^{(r)}(u)-\tilde{P}_{k}(u)\right| d u \\
& \left.\quad=\int_{0}^{x} \frac{(x-u)^{r-1}}{w_{r / 2+\alpha, r / 2+\beta}(u)} w_{r / 2+\alpha, r / 2+\beta}(u) \mid f^{(r)}(u)-\tilde{P}_{k}(u)\right) \mid d u \\
& \quad \leq c A_{q}(x) W_{r, p},
\end{aligned}
$$

where $q:=p /(p-1)$,

$$
A_{q}(x):=\left(\int_{0}^{x}\left(\frac{(x-u)^{r-1}}{w_{r / 2+\alpha, r / 2+\beta}(u)}\right)^{q} d u\right)^{1 / q}, \quad \text { if } \quad q<\infty
$$

and

$$
A_{\infty}(x):=\sup _{u \in[0, x]}\left(\frac{(x-u)^{r-1}}{w_{r / 2+\alpha, r / 2+\beta}(u)}\right)
$$

Now, since

$$
\frac{(x-u)^{r-1}}{w_{r / 2+\alpha, r / 2+\beta}(u)} \leq \frac{(x-u)^{r-1}}{(1-u)^{r / 2+\alpha}} \leq(1-u)^{r / 2-\alpha-1}
$$

we have

$$
A_{q}^{q}(x) \leq \int_{0}^{x}(1-u)^{q(r / 2-\alpha-1)} d u \quad \text { and } \quad A_{\infty}(x) \leq \sup _{u \in[0, x]}(1-u)^{r / 2-\alpha-1} .
$$

If $q<\infty$ and $q(r / 2-\alpha-1)>-1$, then

$$
A_{q}^{q}(x) \leq \int_{0}^{1}(1-u)^{q(r / 2-\alpha-1)} d u=c
$$

which yields

$$
\left\|f-P_{k+r}\right\|_{L_{\infty}[0,1]} \leq c W_{r, p}
$$

and hence

$$
\left\|w_{\alpha, \beta}\left(f-P_{k+r}\right)\right\|_{L_{p}[0,1]} \leq c W_{r, p}\left\|w_{\alpha, \beta}\right\|_{L_{p}[0,1]} \leq c W_{r, p}
$$

where we used the fact that $\alpha \in J_{p}$. Similarly, (3.16) holds if $q=\infty(p=1)$ and $r / 2-\alpha-1 \geq 0$.

If $q<\infty$ and $q(r / 2-\alpha-1)<-1$, then

$$
A_{q}^{q}(x) \leq c(1-x)^{q(r / 2-\alpha-1)+1},
$$


and so, recalling that $x \geq 0$, we have

$$
w_{\alpha, \beta}(x) A_{q}(x) \leq c(1-x)^{r / 2-1 / p} .
$$

Hence,

$$
\left\|w_{\alpha, \beta}\left(f-P_{k+r}\right)\right\|_{L_{p}[0,1]} \leq c\left\|w_{\alpha, \beta} A_{q}\right\|_{L_{p}[0,1]} W_{r, p} \leq c W_{r, p} .
$$

Similarly, one shows that (3.17) holds if $q=\infty(p=1)$ and $r / 2-\alpha-1<0$.

It remains to consider the case $q<\infty$ and $q(r / 2-\alpha-1)=-1$. We have

$$
A_{q}^{q}(x) \leq \int_{0}^{x}(1-u)^{-1} d u=c|\ln (1-x)|
$$

and so

$$
w_{\alpha, \beta}(x) A_{q}(x) \leq c(1-x)^{\alpha}|\ln (1-x)|^{1 / q} .
$$

For $p<\infty$, since $\alpha p>-1$, we have

$$
\left\|w_{\alpha, \beta} A_{q}\right\|_{L_{p}[0,1]}^{p} \leq c \int_{0}^{1}(1-x)^{\alpha p}|\ln (1-x)|^{p-1} d x<c .
$$

Finally, if $p=\infty$, then $q=1$ and $\alpha=r / 2>0$, and so $\left\|w_{\alpha, \beta} A_{1}\right\|_{L_{\infty}[0,1]}<c$. Hence, (3.17) holds in this case as well.

Similarly, one shows that

$$
\left\|w_{\alpha, \beta}\left(f-P_{k+r}\right)\right\|_{L_{p}[-1,0]} \leq c W_{r, p}
$$

and the proof is complete.

\section{Direct estimates: proof of Theorems 1.4 and 1.5}

The following lemma is [13, Corollary 4.4] with $r=0$.

jacklemma

Lemma 4.1. Let $k \in \mathbb{N}, \alpha \geq 0, \beta \geq 0$ and $f \in L_{p}^{\alpha, \beta}, 0<p \leq \infty$. Then, there exists $N \in \mathbb{N}$ depending on $k, p, \alpha$ and $\beta$, such that for every $n \geq N$ and $0<\vartheta \leq 1$, there is a polynomial $P_{n} \in \mathbb{P}_{n}$ satisfying

$$
\left\|w_{\alpha, \beta}\left(f-P_{n}\right)\right\|_{p} \leq c \omega_{k, 0}^{* \varphi}(f, \vartheta / n)_{\alpha, \beta, p} \leq c \omega_{k, 0}^{\varphi}(f, \vartheta / n)_{\alpha, \beta, p}
$$

and

$$
n^{-k}\left\|w_{\alpha, \beta} \varphi^{k} P_{n}^{(k)}\right\|_{p} \leq c \omega_{k, 0}^{* \varphi}(f, \vartheta / n)_{\alpha, \beta, p} \leq c \omega_{k, 0}^{\varphi}(f, \vartheta / n)_{\alpha, \beta, p}
$$

where constants $c$ depend only on $k, p, \alpha, \beta$ and $\vartheta$.

Proof of Theorem 1.4. Estimate (1.5) immediately follows from Lemma 4.1 for $n \geq N$. For $k \leq n<N$, (1.5) follows from Corollary 3.2 with $\theta:=1 / N$, since

$$
E_{n}(f)_{\alpha, \beta, p} \leq E_{k}(f)_{\alpha, \beta, p} \leq c \omega_{k, 0}^{\varphi}(f, 1 / N)_{\alpha, \beta, p} \leq c \omega_{k, 0}^{\varphi}(f, 1 / n)_{\alpha, \beta, p} .
$$


Remark 4.2. In the case $1 \leq p \leq \infty$, it was shown by Ky [14, Theorem 4] (see also Luther and Russo [15]) that if $\alpha, \beta \geq 0$, then

$$
E_{n}(f)_{w_{\alpha, \beta}, p} \leq c \omega_{\varphi}^{k}(f, 1 / n)_{w_{\alpha, \beta}, p}, \quad n \geq n_{0} .
$$

By virtue of [13, (2.2)], we have, for $1 \leq p \leq \infty$,

$$
\omega_{k, r}^{\varphi}\left(f^{(r)}, t\right)_{\alpha, \beta, p} \sim \omega_{\varphi}^{k}\left(f^{(r)}, t\right)_{w_{\alpha, \beta} \varphi^{r}, p}, \quad 0<t \leq t_{0} .
$$

Thus, in the case $1 \leq p \leq \infty$, (1.5) with $n \geq n_{0}$ follows from (4.1). We also remark that, even though (4.1) was stated with $n_{0}=k$ in [14], the proof used [5, Theorem 6.1.1] where $0<t \leq t_{0}$, and so was only justified for sufficiently large $n$.

Proof of Theorem 1.5. The case $r=0$ is Theorem 1.4. Thus we may assume that $r \geq 1$. It follows by [5, Theorem 8.2.1 and (6.3.2)] that, for $n \geq n_{0}$,

estim

$$
\begin{aligned}
E_{n}(f)_{w_{\alpha, \beta}, p} & \leq c \int_{0}^{1 / n}\left(\Omega_{\varphi}^{k+r}(f, t)_{w_{\alpha, \beta}, p} / t\right) d t \\
& \leq c \int_{0}^{1 / n} t^{r-1} \Omega_{\varphi}^{k}\left(f^{(r)}, t\right)_{w_{\alpha, \beta} \varphi^{r}, p} d t \\
& \leq \frac{c}{n^{r}} \Omega_{\varphi}^{k}\left(f^{(r)}, 1 / n\right)_{w_{\alpha, \beta} \varphi^{r}, p} \leq \frac{c}{n^{r}} \omega_{\varphi}^{k}\left(f^{(r)}, 1 / n\right)_{w_{\alpha, \beta} \varphi^{r}, p},
\end{aligned}
$$

and so (1.5) follows by (4.2). For $k+r \leq n<n_{0}$, (1.5) immediately follows from Theorem 3.4 with $\theta:=1 / n_{0}$, as above. This completes the proof.

\section{Inverse theorem: proof of Theorem 1.6}

We first prove this theorem in the case $r \geq 1$.

For the proof we need the following fundamental inequality (see [7,17] as well as [5. (8.1.3)]): given $\alpha, \beta \in J_{p}, 1 \leq p \leq \infty$, we have

$$
\left\|w_{\alpha, \beta} \varphi^{r} p_{n}^{(r)}\right\|_{p} \leq c(r, p, \alpha, \beta) n^{r}\left\|w_{\alpha, \beta} p_{n}\right\|_{p}, \quad p_{n} \in \mathbb{P}_{n} .
$$

Let $f \in L_{p}^{\alpha, \beta}$ and let $P_{n} \in \mathbb{P}_{n}$ be a polynomial of best approximation of $f$ in $L_{p}^{\alpha, \beta}$. Then $E_{n}(f)_{w_{\alpha, \beta}, p}=\left\|w_{\alpha, \beta}\left(f-P_{n}\right)\right\|_{p}, n \geq 1$.

Throughout the proof, we often use the estimate

$$
\begin{aligned}
& \sum_{j=l}^{m}\left(2^{j} N\right)^{\nu} E_{2^{j} N}(f)_{w_{\alpha, \beta}, p} \\
& \leq\left(1+2^{\nu}\right) \sum_{j=l}^{m-1}\left(2^{j} N\right)^{\nu} E_{2^{j} N}(f)_{w_{\alpha, \beta}, p} \\
& \leq\left(1+2^{\nu}\right) 2^{\nu} \sum_{j=l}^{m-1} \sum_{n=2^{j-1} N+1}^{2^{j} N} n^{\nu-1} E_{n}(f)_{w_{\alpha, \beta}, p} \\
& =\left(1+2^{\nu}\right) 2^{\nu} \sum_{n=2^{l-1} N+1}^{2^{m-1} N} n^{\nu-1} E_{n}(f)_{w_{\alpha, \beta}, p},
\end{aligned}
$$

where $\nu \geq 1$ and $1 \leq l<m$, which is also valid if $m=\infty$. 
We represent $f$ as the telescopic series

teles

$$
f=P_{k+r}+\left(P_{N}-P_{k+r}\right)+\sum_{j=0}^{\infty}\left(P_{2^{j+1} N}-P_{2^{j} N}\right)=: P_{k+r}+Q+\sum_{j=0}^{\infty} Q_{j} .
$$

Since

$$
\left\|w_{\alpha, \beta} Q_{j}\right\|_{p} \leq\left\|w_{\alpha, \beta}\left(P_{2^{j+1} N}-f\right)\right\|_{p}+\left\|w_{\alpha, \beta}\left(f-P_{2^{j} N}\right)\right\|_{p} \leq c E_{2^{j} N}(f)_{w_{\alpha, \beta}, p},
$$

we have by virtue of (5.1) and (1.7), for each $1 \leq \nu \leq r$,

$$
\begin{aligned}
\sum_{j=0}^{\infty}\left\|w_{\alpha, \beta} \varphi^{\nu} Q_{j}^{(\nu)}\right\|_{p} & \leq c \sum_{j=0}^{\infty}\left(2^{j+1} N\right)^{\nu} E_{2^{j} N}(f)_{w_{\alpha, \beta}, p} \\
& \leq c N^{\nu} E_{N}(f)_{w_{\alpha, \beta}, p}+c \sum_{n=N+1}^{\infty} n^{\nu-1} E_{n}(f)_{w_{\alpha, \beta}, p}<\infty
\end{aligned}
$$

By the same argument as in the proof of [12, Theorem 9.1], it follows that almost everywhere $f(x)$ is identical with a function possessing an absolutely continuous derivative of order $(r-1)$ and $f^{(r)} \in L_{p}[-1+\varepsilon, 1-\varepsilon]$, for any $\varepsilon>0$. In particular, differentiation of (5.3) is justified, and $f \in \mathbb{B}_{p}^{r}\left(w_{\alpha, \beta}\right)$.

By [13, Lemma 4.1], since $r / 2+\alpha \geq 0$ and $r / 2+\beta \geq 0$, we have

$$
\omega_{k, r}^{\varphi}\left(Q_{j}^{(r)}, t\right)_{\alpha, \beta, p} \leq c\left\|w_{\alpha, \beta} \varphi^{r} Q_{j}^{(r)}\right\|_{p}
$$

and

$$
\omega_{k, r}^{\varphi}\left(Q_{j}^{(r)}, t\right)_{\alpha, \beta, p} \leq c t^{k}\left\|w_{\alpha, \beta} \varphi^{r+k} Q_{j}^{(r+k)}\right\|_{p} .
$$

Hence, by (5.1) and (5.4) we obtain

$$
\omega_{k, r}^{\varphi}\left(Q_{j}^{(r)}, t\right)_{\alpha, \beta, p} \leq c\left(2^{j+1} N\right)^{r}\left\|w_{\alpha, \beta} Q_{j}\right\|_{p} \leq c\left(2^{j} N\right)^{r} E_{2^{j} N}(f)_{w_{\alpha, \beta}, p}
$$

and

$$
\omega_{k, r}^{\varphi}\left(Q_{j}^{(r)}, t\right)_{\alpha, \beta, p} \leq c t^{k}\left(2^{j+1} N\right)^{r+k}\left\|w_{\alpha, \beta} Q_{j}\right\|_{p} \leq c t^{k}\left(2^{j} N\right)^{r+k} E_{2^{j} N}(f)_{w_{\alpha, \beta}, p} .
$$

Denoting $J:=\min \left\{j \in \mathbb{N}_{0}: 2^{-j} \leq N t\right\}$ (note that $2^{-J} \leq N t<2^{-J+1}$ if $J \geq 1$, and $N t \geq 1$ if $J=0$ ) we now have by (5.2)

$$
\begin{aligned}
\omega_{k, r}^{\varphi}\left(\sum_{j=J+1}^{\infty} Q_{j}^{(r)}, t\right)_{\alpha, \beta, p} & \leq c \sum_{j=J+1}^{\infty} \omega_{k, r}^{\varphi}\left(Q_{j}^{(r)}, t\right)_{\alpha, \beta, p} \\
& \leq c \sum_{j=J+1}^{\infty}\left(2^{j} N\right)^{r} E_{2^{j} N}(f)_{w_{\alpha, \beta}, p} \\
& \leq c \sum_{n=2^{J} N+1}^{\infty} n^{r-1} E_{n}(f)_{w_{\alpha, \beta}, p} \\
& \leq c \sum_{n>\max \{N, 1 / t\}} n^{r-1} E_{n}(f)_{w_{\alpha, \beta}, p}
\end{aligned}
$$


since $2^{J} N+1>\max \{N, 1 / t\}$. Now, if $J \geq 2$, then (5.2) implies

app2

$$
\begin{aligned}
& \omega_{k, r}^{\varphi}\left(\sum_{j=0}^{J} Q_{j}^{(r)}, t\right)_{\alpha, \beta, p} \leq c t^{k} \sum_{j=0}^{J}\left(2^{j} N\right)^{r+k} E_{2^{j} N}(f)_{w_{\alpha, \beta}, p} \\
& \leq c t^{k} N^{r+k} E_{N}(f)_{w_{\alpha, \beta}, p}+c t^{k} \sum_{n=N+1}^{2^{J-1} N} n^{r+k-1} E_{n}(f)_{w_{\alpha, \beta}, p} \\
& \leq c t^{k} N^{r+k} E_{N}(f)_{w_{\alpha, \beta}, p}+c t^{k} \sum_{N \leq n \leq \max \{N, 1 / t\}} n^{k+r-1} E_{n}(f)_{w_{\alpha, \beta}, p},
\end{aligned}
$$

where we used the fact that $2^{J-1} N \leq \max \{N, 1 / t\}$. If $J=0$ or 1 , then we have

$$
\omega_{k, r}^{\varphi}\left(\sum_{j=0}^{J} Q_{j}^{(r)}, t\right)_{\alpha, \beta, p} \leq c t^{k} N^{r+k} E_{N}(f)_{w_{\alpha, \beta}, p},
$$

and so the last estimate in (5.6) is valid in this case as well.

Finally, if $N \geq k+r$, then

$$
\begin{aligned}
\omega_{k, r}^{\varphi}\left(P_{k+r}^{(r)}+Q^{(r)}, t\right)_{\alpha, \beta, p} & =\omega_{k, r}^{\varphi}\left(Q^{(r)}, t\right)_{\alpha, \beta, p} \leq c t^{k}\left\|w_{\alpha, \beta} \varphi^{k+r} Q^{(k+r)}\right\|_{p} \\
& \leq c t^{k} N^{r+k}\left\|w_{\alpha, \beta} Q\right\|_{p} \leq c t^{k} N^{r+k} E_{k+r}(f)_{w_{\alpha, \beta}, p}
\end{aligned}
$$

and if $N<k+r$, then $\omega_{k, r}^{\varphi}\left(P_{k+r}^{(r)}+Q^{(r)}, t\right)_{\alpha, \beta, p}=0$, so that we don't need (5.7).

Combining (5.5)-(5.7) and using the fact that, if $N \geq k+r$, then $E_{N}(f)_{w_{\alpha, \beta}, p} \leq$ $E_{k+r}(f)_{w_{\alpha, \beta}, p}$ and, if $N<k+r$, then the first term in the last inequality in (5.6) can be absorbed by the second term in that inequality, we obtain (1.8), and our proof is complete in the case $r \geq 1$.

Suppose now that $r=0$. We represent $f$ as

teleszero

$$
f=P_{k}+Q+\sum_{j=0}^{J} Q_{j}+\left(f-P_{2^{J+1} N}\right),
$$

where $Q:=P_{N}-P_{k}$ and $Q_{j}:=P_{2^{j+1} N}-P_{2^{j} N}$, and estimate the last term. We have

$$
\left\|w_{\alpha, \beta}\left(f-P_{2^{J+1} N}\right)\right\|_{p} \leq c E_{2^{J+1} N}(f)_{w_{\alpha, \beta}, p},
$$

and in the case $J=0$ or 1 , we use the fact that $N t \geq c$, to conclude

$$
E_{2^{J+1} N}(f)_{w_{\alpha, \beta}, p} \leq E_{N}(f)_{w_{\alpha, \beta}, p}=N^{k} t^{k}(N t)^{-k} E_{N}(f)_{w_{\alpha, \beta}, p} \leq c(N) t^{k} E_{N}(f)_{w_{\alpha, \beta}, p} .
$$

If $J \geq 2$, we recall that $2^{J-1} N<1 / t \leq 2^{J} N$, so that $\max \{N, 1 / t\}=1 / t$, and write

$$
\begin{aligned}
E_{2^{J+1} N}(f)_{w_{\alpha, \beta}, p} & \leq\left(2^{J-2} N\right)^{-1} \sum_{n=2^{J-2} N+1}^{2^{J-1} N} E_{n}(f)_{w_{\alpha, \beta}, p} \\
& \leq\left(2^{J-2} N\right)^{-k} \sum_{n=2^{J-2} N+1}^{2^{J-1} N} n^{k-1} E_{n}(f)_{w_{\alpha, \beta}, p} \\
& \leq 4^{k} t^{k} \sum_{N \leq n<1 / t} n^{k-1} E_{n}(f)_{w_{\alpha, \beta}, p} .
\end{aligned}
$$

It now remains to apply (5.6) and (5.7) with $r=0$, in order to complete the proof of (1.8) in the case $r=0$. 


\section{Appendix}

The following sharp Marchaud inequality was proved in [2].

Theorem 6.1 ([2, Theorem 7.5]). For $m \in \mathbb{N}, 1<p<\infty$ and $\alpha, \beta \in J_{p}$, we have

$$
K_{m, \varphi}\left(f, t^{m}\right)_{w_{\alpha, \beta}, p} \leq C t^{m}\left(\int_{t}^{1} \frac{K_{m+1, \varphi}\left(f, u^{m+1}\right)_{w_{\alpha, \beta}, p}^{q}}{u^{m q+1}} d u+E_{m}(f)_{w_{\alpha, \beta}, p}^{q}\right)^{1 / q}
$$

and

$$
K_{m, \varphi}\left(f, t^{m}\right)_{w_{\alpha, \beta}, p} \leq C t^{m}\left(\sum_{n<1 / t} n^{q m-1} E_{n}(f)_{w_{\alpha, \beta}, p}^{q}\right)^{1 / q}
$$

where $q=\min (2, p)$.

Corollary 6.2. For $1<p<\infty, r \in \mathbb{N}_{0}, m \in \mathbb{N}, r / 2+\alpha \geq 0, r / 2+\beta \geq 0$ and $f \in \mathbb{B}_{p}^{r}\left(w_{\alpha, \beta}\right)$, we have

$$
\omega_{m, r}^{\varphi}\left(f^{(r)}, t\right)_{\alpha, \beta, p} \leq C t^{m}\left(\int_{t}^{1} \frac{\omega_{m+1, r}^{\varphi}\left(f^{(r)}, u\right)_{\alpha, \beta, p}^{q}}{u^{m q+1}} d u+E_{m}\left(f^{(r)}\right)_{w_{\alpha, \beta} \varphi^{r}, p}^{q}\right)^{1 / q}
$$

and

$$
\omega_{m, r}^{\varphi}\left(f^{(r)}, t\right)_{\alpha, \beta, p} \leq C t^{m}\left(\sum_{n<1 / t} n^{q m-1} E_{n}\left(f^{(r)}\right)_{w_{\alpha, \beta} \varphi^{r}, p}^{q}\right)^{1 / q},
$$

where $q=\min (2, p)$.

The following sharp Jackson inequality was proved in [3].

Theorem 6.3 ([3, Theorem 6.2]). For $1<p<\infty, \alpha, \beta \in J_{p}$ and $m \in \mathbb{N}$, we have

$$
2^{-n m}\left(\sum_{j=j_{0}}^{n} 2^{m j s} E_{2^{j}}(f)_{w_{\alpha, \beta}, p}^{s}\right)^{1 / s} \leq C K_{m, \varphi}\left(f, 2^{-n m}\right)_{w_{\alpha, \beta}, p}
$$

and

$$
2^{-n m}\left(\sum_{j=j_{0}}^{n} 2^{m j s} K_{m+1, \varphi}\left(f, 2^{-j(m+1)}\right)_{w_{\alpha, \beta}, p}^{s}\right)^{1 / s} \leq C K_{m, \varphi}\left(f, 2^{-n m}\right)_{w_{\alpha, \beta}, p},
$$

where $2^{j_{0}} \geq m$ and $s=\max (p, 2)$.

Corollary 6.4. For $1<p<\infty, r \in \mathbb{N}_{0}, m \in \mathbb{N}, r / 2+\alpha \geq 0, r / 2+\beta \geq 0$ and $f \in \mathbb{B}_{p}^{r}\left(w_{\alpha, \beta}\right)$, we have

$$
2^{-n m}\left(\sum_{j=j_{0}}^{n} 2^{m j s} E_{2^{j}}\left(f^{(r)}\right)_{w_{\alpha, \beta} \varphi^{r}, p}^{s}\right)^{1 / s} \leq C \omega_{m, r}^{\varphi}\left(f^{(r)}, 2^{-n}\right)_{\alpha, \beta, p}
$$

and

$$
2^{-n m}\left(\sum_{j=j_{0}}^{n} 2^{m j s} \omega_{m+1, r}^{\varphi}\left(f^{(r)}, 2^{-j}\right)_{\alpha, \beta, p}^{s}\right)^{1 / s} \leq C \omega_{m, r}^{\varphi}\left(f^{(r)}, 2^{-n}\right)_{\alpha, \beta, p},
$$

where $2^{j_{0}} \geq m$ and $s=\max (p, 2)$. 
Corollary 6.5. For $1<p<\infty, r \in \mathbb{N}_{0}, m \in \mathbb{N}, r / 2+\alpha \geq 0, r / 2+\beta \geq 0$ and $f \in \mathbb{B}_{p}^{r}\left(w_{\alpha, \beta}\right)$, we have

$$
t^{m}\left(\int_{t}^{1 / m} \frac{\omega_{m+1, r}^{\varphi}\left(f^{(r)}, u\right)_{\alpha, \beta, p}^{s}}{u^{m s+1}} d u\right)^{1 / s} \leq C \omega_{m, r}^{\varphi}\left(f^{(r)}, t\right)_{\alpha, \beta, p}, \quad 0<t \leq 1 / m,
$$

where $s=\max (p, 2)$.

\section{References}

$\mathrm{b}$

dd

ddt

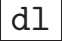

$\mathrm{dt}$

$\mathrm{sh}$

kha

k-at 8

k-sing

kls-umzh

kls1

$\mathrm{kls}$

sam

ky

$\operatorname{lr}$

$\mathrm{pp}$

pot

[1] Yu. A. Brudnyi, On a theorem of local approximations, Kazan. Gos. Univ. Učen. Zap 12 (1964), no. 6, 43-49 (Russian).

[2] F. Dai and Z. Ditzian, Littlewood-Paley theory and a sharp Marchaud inequality, Acta Sci. Math. (Szeged) 71 (2005), no. 1-2, 65-90.

[3] F. Dai, Z. Ditzian, and S. Tikhonov, Sharp Jackson inequalities, J. Approx. Theory 151 (2008), no. 1, 86-112.

[4] R. A. DeVore and G. G. Lorentz, Constructive approximation, A Series of Comprehensive Studies in Mathematics, vol. 303, Springer-Verlag, New York, 1993.

[5] Z. Ditzian and V. Totik, Moduli of smoothness, Springer Series in Computational Mathematics, vol. 9, Springer-Verlag, New York, 1987.

[6] V. K. Dzyadyk and I. A. Shevchuk, Theory of Uniform Approximation of Functions by Polynomials, Walter de Gruyter, Berlin, 2008.

[7] B. A. Khalilova, Certain estimates for polynomials, Izv. Akad. Nauk Azerbaĭdžan. SSR Ser. Fiz.-Tehn. Mat. Nauk 2 (1974), 46-55 (in Russian, with Azerbaijani and English summaries).

[8] K. A. Kopotun, On $K$-monotone polynomial and spline approximation in $L_{p}, 0<$ $p<\infty$ (quasi)norm, Approximation theory VIII, Vol. 1 (College Station, TX, 1995), Ser. Approx. Decompos., vol. 6, World Sci. Publ., River Edge, NJ, 1995, pp. 295-302.

[9] _ Polynomial approximation with doubling weights having finitely many zeros and singularities, J. Approx. Theory 198 (2015), 24-62.

[10] K. A. Kopotun, D. Leviatan, and I. A. Shevchuk, Are the degrees of the best (co)convex and unconstrained polynomial approximations the same? II, Ukraïn. Mat. Zh. 62 (2010), no. 3, 369-386; English transl., Ukrainian Math. J. 62 (2010), no. 3, 420-440.

[11] _ New moduli of smoothness, Publ. l'Inst. Math. Serbian Academy of Sciences and Arts of Belgrade 96(110) (2014), 169-180.

[12] _ New moduli of smoothness: Weighted DT moduli revisited and applied, Constr. Approx. 42 (2015), 129-159.

[13] _ On moduli of smoothness with Jacobi weights, Ukrainian Math. J.

[14] N. X. Ky, On approximation of functions by polynomials with weight, Acta Math. Hungar. 59 (1992), no. 1-2, 49-58.

[15] U. Luther and M. G. Russo, Boundedness of the Hilbert transformation in some weighted Besov type spaces, Integral Equations Operator Theory 36 (2000), no. 2, 220-240.

[16] P. P. Petrushev and V. A. Popov, Rational approximation of real functions, Encyclopedia of Mathematics and its Applications, vol. 28, Cambridge University Press, Cambridge, 1987.

[17] M. K. Potapov, Some inequalities for polynomials and their derivatives, (in Russian), Vestnik Moscow Univ. 2 (1960), 10-19. 
[18] E. A. Storozhenko, On approximation by algebraic polynomials of functions in the class $L_{p}, 0<p<1$, Izv. Akad. Nauk USSR 41 (1977), 652-662 (in Russian).

[19] H. Whitney, On functions with bounded $n^{\text {th }}$ differences, J. Math. Pure et Appl. 36 (1957), 67-95. 